\title{
Spin Hall mode in a trapped thermal Rashba gas
}

\author{
J. Armaitis, ${ }^{1, *}$ J. Ruseckas, ${ }^{1}$ H. T. C. Stoof, ${ }^{2,3}$ and R. A. Duine ${ }^{2,3,4}$ \\ ${ }^{1}$ Institute of Theoretical Physics and Astronomy, Vilnius University, Sauletekio Avenue 3, LT-10222 Vilnius, Lithuania \\ ${ }^{2}$ Institute for Theoretical Physics, Utrecht University, Princetonplein 5, 3584 CC Utrecht, Netherlands \\ ${ }^{3}$ Center for Extreme Matter and Emergent Phenomena, Utrecht University, Princetonplein 5, 3584 CC Utrecht, Netherlands \\ ${ }^{4}$ Department of Applied Physics, Eindhoven University of Technology, P.O. Box 513, 5600 MB Eindhoven, Netherlands
}

(Received 1 September 2017; published 20 November 2017)

\begin{abstract}
We theoretically investigate a two-dimensional harmonically trapped gas of identical atoms with Rashba spin-orbit coupling and no interatomic interactions. In analogy with the spin Hall effect in uniform space, the gas exhibits a spin Hall mode. In particular, in response to a displacement of the center of mass of the system, spin-dipole moment oscillations occur. We determine the properties of these oscillations exactly and find that their amplitude strongly depends on the spin-orbit-coupling strength and the quantum statistics of the particles.
\end{abstract}

DOI: 10.1103/PhysRevA.96.053625

\section{INTRODUCTION}

Collective behavior provides an important pathway towards the measurement of various physical properties in a multitude of systems. In particular, material properties pertaining to transport often leave an imprint in the collective motion of the system. For example, the charge-carrier density can be determined by observing the Hall response [1]. Various magnetic oscillations allow us to measure the effective mass of carriers, as well as to quantify the level of disorder in the material [2]. The speed of sound in a material allows us to access information about such mechanical properties as shear modulus, density, and compressibility [3]. Recently, vorticity has been identified as a hallmark of viscous electron transport in graphene $[4,5]$.

When it comes to ultracold atomic gas systems, collective modes have been at the center of the field since the very first experiments [6,7]. By "collective modes" in this context it is meant that the system is perturbed as a whole. The response to this perturbation is typically deduced from measurements that probe the full system and not its constituents, even in the absence of interparticle interactions. Arguably, the simplest of such modes is the center-of-mass oscillation (also known as the dipole mode) of the whole cloud of atoms in a harmonic trap. Kohn's theorem states that this mode does not decay and is not affected by interactions and that its frequency equals that of the trap [8,9]. This theorem does not apply in the presence of spin-orbit coupling (SOC). SOC breaks Galilean invariance [10-13], as demonstrated by the altered frequency of the dipole mode [14,15]. Another case when Kohn's theorem does not apply is an out-of-phase oscillation of two interacting species of atoms in the same trap, i.e., a so-called (pseudo)spin-dipole oscillation. To probe this mode, a species-dependent force displaces the clouds of the two species with respect to each other while keeping the center of mass of the whole system at the bottom of the trap. Such oscillations have been observed in various experiments with bosons [16-19], fermions [20,21], and mixtures of bosons and fermions [22-24].

A similar excitation in response to a spin-independent force known as the intrinsic spin Hall effect has been central

\footnotetext{
*jogundas.armaitis@tfai.vu.lt
}

to the field of spintronics [25,26]. There, a transverse spin current is generated in response to a longitudinal charge current in a uniform system. This occurs generically due to the presence of (Rashba [27] or Dresselhaus [28]) SOC since the spin of different momentum states precesses differently in response to a spin-independent force. Building upon this knowledge, we analyze such a situation in the presence of a trapping potential, where one expects a similar response to occur (Fig. 1). Contrary to the spintronics nomenclature, in the ultracold-atom literature, the spin Hall effect almost [29] always refers to the two eigenstates of a system with one-dimensional (also known as equal Rashba-Dresselhaus) SOC experiencing opposite transverse forces in the absence of spin precession [30,31]. Spin is correspondingly conserved in one direction in these ultracold-atom experiments. However, this does not have to be the case in general. Indeed, spin is typically not conserved in solid-state spintronics experiments [26].

Several different schemes for inducing two-dimensional SOC in ultracold-atom systems have been proposed [32-35], and at least one of them has been experimentally realized [36]. Hence, we are motivated to investigate the collective modes of a harmonically confined gas in the presence of a twodimensional SOC. Furthermore, since all the experimentally realistic two-dimensional SOC schemes involve some degree of heating $[10,11,37]$, at least the early experiments are likely to operate in the thermal-gas regime. Therefore, we study quantum degenerate thermal, i.e., noncondensed, fermions and bosons. In this regime energy scales set by various scattering processes (interparticle interactions) can be made small compared to the thermal energy, e.g., by employing a Feshbach resonance [38], allowing us to neglect interaction effects in this study. Moreover, we do not consider the constant Zeeman terms that break the time-reversal symmetry and thereby lead to an anomalous Hall effect [39]. This allows us to stay in the regime where the transverse Hall response exists only in the spin channel.

Our main finding is that a collective mode in the trap analogous to the spin Hall response in the uniform system is indeed present. We call this mode the spin Hall mode. The amplitude of this response depends in a nontrivial way on the SOC strength and is different for Bose and Fermi particles (see Figs. 4 and 6 below). 


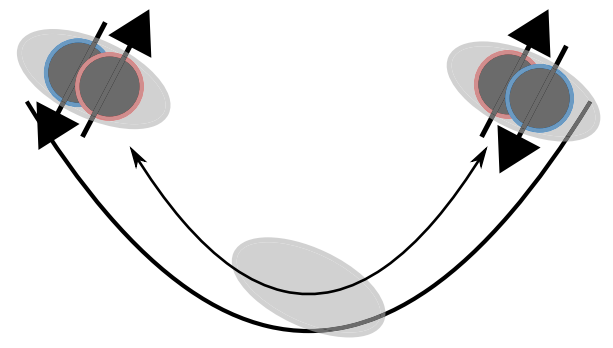

FIG. 1. Spin Hall mode: due to the spin Hall effect, a Rashba spin-orbit-coupled system (gray ellipsoid) in a harmonic trap (thick parabola) exhibits oscillations of the transverse spin-dipole moment in response to a displacement away from the bottom of the trap. During these oscillations the whole cloud swings back and forth along the axis of displacement, as indicated by the thin arrow. More importantly, the transverse spin-dipole moment also oscillates, whereas the total spin of the cloud remains zero. This is visualized by the alternating separation of the average positions of the spin-up (red) and spin-down (blue) particles. The spin quantization axis is perpendicular to the spin-orbit-coupling plane, whereas the spinseparation axis is perpendicular to both the spin axis and the direction of displacement.

This paper is organized as follows. In Sec. II we present the problem and introduce the notation. We treat the weakSOC and strong-SOC limits analytically for the lowest-lying states in Sec. III. In Sec. IV we present an exact numerical treatment of both bosonic and fermionic degenerate gases at nonzero temperature. Finally, Sec. V summarizes our results and provides some directions for future work.

\section{THEORETICAL FRAMEWORK}

\section{A. System}

The Hamiltonian of our system

$$
H=H_{K}+H_{R}+H_{T}
$$

consists of the kinetic-energy term $H_{K}$, the Rashba SOC term $H_{R}$, and the harmonic trapping potential $H_{T}$. It is assumed from the outset that a steep trapping potential in the $z$ direction dominates all other energy scales, allowing us to concentrate on the dynamics in the $x-y$ plane. It is convenient to treat the problem in units where the reduced Planck constant, particle mass, and trap frequency are set to unity, $\hbar=M=\omega_{T}=1$. In these units,

$$
\begin{gathered}
H_{K}=\boldsymbol{p}^{2} / 2=\left(p_{x}^{2}+p_{y}^{2}\right) / 2, \\
H_{T}=\boldsymbol{x}^{2} / 2=\left(x^{2}+y^{2}\right) / 2,
\end{gathered}
$$

where vectors are denoted by the bold font, $\boldsymbol{x}$ is a vector of coordinate operators, and $\boldsymbol{p}$ is a vector of momentum operators. We keep identity matrices in spin space implicit throughout the article. The Rashba SOC Hamiltonian is

$$
H_{R}=v\left(\sigma_{x} p_{y}-\sigma_{y} p_{x}\right)
$$

where $v$ is the Rashba SOC strength and $\sigma_{i}$ are the Pauli matrices. Using polar coordinates in the plane, $p_{x}=p \cos \theta$ and $p_{y}=p \sin \theta$, as well as introducing the spin raising and lowering operators

$$
\begin{aligned}
\sigma_{x} & =\sigma_{+}+\sigma_{-}, \\
i \sigma_{y} & =\sigma_{+}-\sigma_{-},
\end{aligned}
$$

the SOC part of the Hamiltonian can be written as

$$
H_{R}=i p v\left(\sigma_{+} e^{-i \theta}-\sigma_{-} e^{i \theta}\right) .
$$

The two eigenspinors of $H_{R}$, namely,

$$
\chi_{ \pm}=\frac{1}{\sqrt{2}}\left(\begin{array}{c} 
\pm i e^{-i \theta} \\
1
\end{array}\right),
$$

depend only on the direction of the momentum in the plane and not on its magnitude. In the absence of the trap, the energies of the two branches corresponding to these eigenspinors are

$$
E_{ \pm}=\frac{p^{2}}{2} \pm p v .
$$

The Hamiltonian $H$ and its various extensions have already been the subject of several investigations. Most of the work has concentrated on the study of Bose-Einstein condensates and their dynamics [40-46]. Some attention has been paid to the interplay between interactions and SOC [47-51], as well as rotations and SOC [52,53]. Moreover, various properties of this system in anisotropic (deformed) trapping potentials have been investigated [54-56]. Regarding dynamics, it was shown that a sudden ramp up of the SOC strength initiates collapse and revival dynamics of the total magnetization for a Fermi gas [57]. Anisotropy in the SOC part of the Hamiltonian leads to chaotic behavior [58].

$H$ in Eq. (1) is also known as the $E \times \varepsilon$ Jahn-Teller Hamiltonian, especially in the context of chemical, molecular, and condensed-matter physics [59]. Larson and coworkers have studied the anomalous Hall effect [60] and the spin Hall effect [61] in that system. In particular, they have shown how the intrinsic asymmetry of the dynamics of the $\mathrm{Li}_{3}$ molecule can be assigned to a spin Hall effect by investigating the evolution of a spin-imbalanced Gaussian wave packet at zero temperature [61]. In contrast, in our paper we concentrate on the observables relevant to ultracold-atom experiments and account for particle statistics and thermal effects.

\section{B. Moments and modes}

In general, one may excite the system using an operator $O_{1}$ and witness the response of the system to this perturbation in the evolution of the expectation values of an operator $\mathrm{O}_{2}$. The response to excitations is intimately related to the eigenmodes of the system.

In a trapped system of ultracold atoms, a natural excitation is a small shift of the center of mass of the system (or, equivalently, the bottom of the trapping potential). Such a perturbation with an infinitesimal amplitude $\left|x_{0}\right| \ll 1$ in a direction $\hat{\boldsymbol{x}}_{0}=\boldsymbol{x}_{0} /\left|\boldsymbol{x}_{0}\right|$ is described by a translation operator

$$
T=1-i \boldsymbol{p} \cdot \boldsymbol{x}_{0} .
$$

In general, this excitation may result in both a longitudinal response (along $\hat{\boldsymbol{x}}_{0}$ ) and a transverse response (along $\hat{\boldsymbol{x}}_{0}^{\perp}$, which is perpendicular to $\hat{\boldsymbol{x}}_{0}$ ). 
Arguably, the simplest observable is the center-of-mass position, namely, $\langle\boldsymbol{x}\rangle$. In a trapped system in the absence of SOC, the response to $T$ is fully described by a single-frequency oscillation in the longitudinal channel of the center-of-mass position of the cloud, $\left\langle\boldsymbol{x} \cdot \hat{\boldsymbol{x}}_{0}\right\rangle$, and is known as the dipole mode or Kohn's mode, as described in the Introduction. In the presence of SOC this mode is significantly modified [14], as will be demonstrated in the subsequent discussion. The center-of-mass response in the transverse channel, $\left\langle\boldsymbol{x} \cdot \hat{\boldsymbol{x}}_{0}^{\perp}\right\rangle$, corresponds to the anomalous Hall effect. It requires a nonzero Berry curvature in momentum space, which can, for instance, be achieved by adding a Zeeman term to our SOC Hamiltonian. An oscillation of the center-of-mass position transverse to the direction of the excitation is therefore called the anomalous Hall mode [39].

Even though expectation values of various other operators are also accessible to ultracold-atom experiments (see, e.g., Ref. [62]), we limit our discussion to the spin-dipole moment $\left\langle x_{i} \sigma_{j}\right\rangle$. As mentioned earlier, it is possible to initialize an ultracold-atom system in a state with a nonzero spin-dipole moment by separating out the two spin states in position space $[16,17,19-24]$. Subsequently, a weakly interacting system in the absence of SOC exhibits spin-dipole oscillations due to harmonic confinement, known as the spin-dipole mode.

From the perspective of spintronics, a transverse spindipole moment or spin accumulation $\left\langle\boldsymbol{x} \cdot \hat{\boldsymbol{x}}_{0}^{\perp} \sigma_{z}\right\rangle$, which emerges in response to a spin-independent perturbation $T$ or voltage, is known as the spin Hall effect [25]. Here we consider exactly such a setup. Namely, we start from a state with a vanishing spin-dipole moment, apply a spin-independent perturbation [Eq. (10)], and subsequently observe oscillations in both the longitudinal center-of-mass position and transverse spin-dipole moment. It is therefore natural to call this collective oscillation the spin Hall mode. In what follows, we investigate this spin Hall mode, focusing on the time dependence of the spin-dipole moment. In particular, we are interested in the magnitude of the spin-dipole moment, which builds up in time.

\section{ANALYTIC RESULTS}

In this section we present an analytic solution for the ground-state response to driving when the SOC is either weak or strong compared to the harmonic trap. Due to time-reversal invariance, all single-particle states are doubly degenerate (Kramer's pairs). Hence, this system has two degenerate many-body ground states, $\left|g_{1}\right\rangle$ and $\left|g_{2}\right\rangle$, regardless of the strength of the SOC. Considering different occupations for these two states breaks the time-reversal symmetry and results in a spurious anomalous Hall effect. We thus assume that these two ground states have equal occupation. Furthermore, we consider statistical mixtures of states throughout the paper and not their quantum-mechanical superpositions, as we focus on thermal excitations.

We follow the unitary evolution of each of the two degenerate ground states of the system after applying an infinitesimal translation operator

$$
T=1-i p_{x} x_{0}
$$

in the $x$ direction. In general, a convenient method to implement unitary time evolution in the Schrödinger picture is projecting the translated state onto the eigenbasis of the full Hamiltonian $H$. To this end, we define the projection operator $\mathcal{P}_{j}=|j\rangle\langle j|$ with respect to the eigenstate $|j\rangle$ of $H$. We are interested in two types of response. First, we consider the center-of-mass position. For a single state $|s\rangle$, the evolution of the average position is given by

$$
\langle x\rangle_{s} \equiv \sum_{j, k} e^{-i\left(E_{k}-E_{j}\right) t}\left\langle s\left|T^{\dagger} \mathcal{P}_{j}^{\dagger} x \mathcal{P}_{k} T\right| s\right\rangle,
$$

and therefore, for the two ground states we have

$$
\langle x\rangle=\frac{1}{2} \sum_{s=g_{1}, g_{2}}\langle x\rangle_{s},
$$

where the factor of $1 / 2$ enters as we consider a mixture of both ground states with equal probability. In the absence of SOC, this expectation value oscillates with the trap frequency $\omega_{T}$. Second, we investigate the spin-dipole moment, the evolution of which for a state $|s\rangle$ is

$$
\left\langle y \sigma_{z}\right\rangle_{s} \equiv \sum_{j, k} e^{-i\left(E_{k}-E_{j}\right) t}\left\langle s\left|T^{\dagger} \mathcal{P}_{j}^{\dagger} y \sigma_{z} \mathcal{P}_{k} T\right| s\right\rangle,
$$

and hence, for the two ground states we have

$$
\left\langle y \sigma_{z}\right\rangle=\frac{1}{2} \sum_{s=g_{1}, g_{2}}\left\langle y \sigma_{z}\right\rangle_{s} .
$$

The spin-dipole moment is excited only by the center-of-mass displacement in the presence of SOC. These two expectation values behave qualitatively differently in the weak- and strongSOC regimes. This is expected since by going from weak to strong SOC, the system undergoes a dimensional reduction from two-dimensional (2D) to effectively one-dimensional (1D) dynamics (see Ref. [63] and references therein for more details). We consider this behavior in detail below.

\section{A. Weak SOC}

In the weak-SOC limit $v \ll 1$ a natural basis for our problem consists of the two-dimensional harmonic oscillator eigenstates $\left|n, m, m_{s}\right\rangle$, where $n$ is the principal quantum number, $m$ is the angular momentum quantum number, and $m_{s}$ is the spin projection quantum number. Nondegenerate perturbation theory can be employed in this case since $m+m_{s}$ is a good quantum number [48]. Since there are two small parameters, $x_{0} \ll 1$ and $v \ll 1$, capturing the response to the lowest order is especially straightforward in this regime. Thus, it is sufficient to consider the second-order energy correction due to $H_{R}$,

$$
\Delta E_{n, m, m_{s}}=\sum_{n^{\prime}, m^{\prime}, m_{s}^{\prime}}^{E_{n, m} \neq E_{n^{\prime}, m^{\prime}}} \frac{\left|\left\langle n, m, m_{s}\left|H_{R}\right| n^{\prime}, m^{\prime}, m_{s}^{\prime}\right\rangle\right|^{2}}{E_{n, m}-E_{n^{\prime}, m^{\prime}}} .
$$

As the translation operator does not commute with the Hamiltonian, it couples the ground state $\left|0,0, m_{s}\right\rangle$ to the states $\left|0, \pm 1, m_{s}\right\rangle$, and the following corrections are required [48]:

$$
\begin{aligned}
& \Delta E_{0,0, \uparrow}=-v^{2}, \quad \Delta E_{0,1, \uparrow}=-2 v^{2}, \quad \Delta E_{0,-1, \uparrow}=0, \\
& \Delta E_{0,0, \downarrow}=-v^{2}, \quad \Delta E_{0,1, \downarrow}=0, \quad \Delta E_{0,-1, \downarrow}=-2 v^{2} .
\end{aligned}
$$



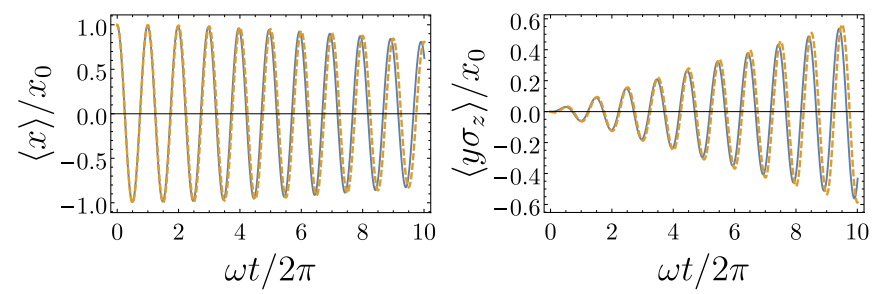

FIG. 2. Center-of-mass position and spin-dipole moment oscillations (spin Hall mode) as a response to displacement of the two ground states along the $x$ direction with the displacement amplitude $x_{0}$ in the weak SOC regime $(v=1 / 10)$. We compare exact numerical results ( $x_{0}=1 / 100$, blue solid line; see Sec. IV for more details) to the analytical results in Eqs. (19) and (20) (infinitesimal $x_{0}$, yellow dashed line) for ten trap periods. Fourier transforming these signals and restoring dimensions yields two peaks at the energies $\hbar \omega_{T} \pm M v^{2}$.

We now take the 2D harmonic oscillator eigenenergies with second-order perturbative corrections due to $H_{R}$ in addition to the unperturbed 2D harmonic oscillator eigenstates. Expanding to the lowest nonvanishing correction, we obtain

$$
\begin{gathered}
\langle x\rangle=x_{0} \cos t \cos v^{2} t, \\
\left\langle y \sigma_{z}\right\rangle=-x_{0} \cos t \sin v^{2} t
\end{gathered}
$$

in this limit. These expressions are unaffected by including corrections to the eigenfunctions up to the second order. Hence, taking a Fourier transform of either $\langle x\rangle$ or $\left\langle y \sigma_{z}\right\rangle$ and restoring dimensions yields two peaks at the energies $\hbar \omega_{T} \pm M v^{2}$. Note, however, that in the limit of weak SOC, increasingly long observation times are required in order to achieve a resolution sufficient to distinguish the two peaks in the Fourier spectrum of this signal. Therefore, it might be more useful to analyze the response directly in the time domain. We compare these analytical results with a numerical simulation for $v=1 / 10$ in Fig. 2. Note that in this weak-SOC regime, center-of-mass oscillations occur around the bottom of the trap with approximately the trap frequency. Here SOC introduces a modulation with a frequency $v^{2}$. The spin-dipole response is shifted by a phase of $\pi / 2$ with respect to the center-of-mass oscillation. The amplitude of the spin-dipole response is the same as the amplitude of the center-of-mass oscillation for the two ground states in this $v \ll 1$ regime. However, note that the spin-dipole moment takes a long time to build up when $v$ is small, practically limiting the magnitude of the response (see Fig. 2).

\section{B. Strong SOC}

In this section we apply the procedure of dimensional reduction pioneered in the field of topological insulators [63] to our system. It allows us to obtain the spectrum and the wave functions explicitly in the strong-SOC regime $1 / v \ll 1$. The spectrum of the system in this limit has already been presented in, e.g., Ref. [41].

In preparation for treating the problem in the strong-SOC regime, it is convenient to write down the Schrödinger equation in momentum space,

$$
\begin{aligned}
& \left\{\frac{p^{2}}{2}+i v p\left(\sigma_{+} e^{-i \theta}-\sigma_{-} e^{i \theta}\right)\right. \\
& \left.-\frac{1}{2}\left(\frac{1}{p} \partial_{p}\left[p \partial_{p}\right]\right)-\frac{1}{2} \frac{1}{p^{2}} \partial_{\theta}^{2}\right\} \Psi=E \Psi .
\end{aligned}
$$

We now make an ansatz

$$
\Psi=\psi_{+}(p) \chi_{+}(\theta) e^{-i m \theta}+\psi_{-}(p) \chi_{-}(\theta) e^{-i m \theta},
$$

which allows us to see that different $m$ states are not coupled since

$$
\begin{aligned}
-\partial_{\theta}^{2}\left(\chi_{ \pm} e^{-i m \theta}\right)= & \left(m[m+1]+\frac{1}{2}\right) \chi_{ \pm} e^{-i m \theta} \\
& -\left(m+\frac{1}{2}\right) e^{-i m \theta} \chi_{\mp} e^{-i m \theta}
\end{aligned}
$$

and

$$
\begin{aligned}
& {\left[\frac{p^{2}}{2}+i v p\left(\sigma_{+} e^{-i \theta}-\sigma_{-} e^{i \theta}\right)\right] \Psi} \\
& \quad=E_{+} \psi_{+} \chi_{+} e^{-i m \theta}+E_{-} \psi_{-} \chi_{-} e^{-i m \theta} .
\end{aligned}
$$

Projecting Eq. (21) to the two branches yields

$$
\begin{aligned}
& E_{ \pm} \psi_{ \pm}-\frac{1}{2}\left(\frac{1}{p} \partial_{p}\left[p \partial_{p} \psi_{ \pm}\right]\right) \\
& +\frac{1}{2 p^{2}}\left[\left(m[m+1]+\frac{1}{2}\right) \psi_{ \pm}-\left(m+\frac{1}{2}\right) \psi_{\mp}\right]=E \psi_{ \pm}
\end{aligned}
$$

where $E_{ \pm}$was defined in Eq. (9). Note that all the considerations up to this point have been exact. Now we specialize to the strong-SOC regime, where the two branches are separated by a large energy gap, save for the $p=0$ point. Relying on this fact, we assume that the upper branch is empty, $\psi_{+}=0$, while the lower branch is described by the wave function

$$
\psi_{-}=f(p) / \sqrt{p} .
$$

The Schrödinger equation for $f(p)$ thus is

$$
E_{-} f-\frac{f}{8 p^{2}}-\frac{1}{2} \partial_{p}^{2} f+\frac{1}{2 p^{2}}\left(m[m+1]+\frac{1}{2}\right) f=E f .
$$

In order to investigate the low-lying states close to the Rashba ring $p=v$, we complete the square,

$$
\begin{aligned}
- & \frac{1}{2} \partial_{p}^{2} f+\left(\frac{p^{2}}{2}-p v+\frac{v^{2}}{2}\right) f-\frac{f}{8 v^{2}}-\frac{v^{2}}{2} f \\
+ & \frac{1}{2 p^{2}}\left(m[m+1]+\frac{1}{2}\right) f=E f,
\end{aligned}
$$

and notice that the first two terms on the left-hand side describe a one-dimensional harmonic oscillator. For the remaining terms, we approximate $p \simeq v$ and obtain the following spectrum:

$$
E_{v m}=\left(v+\frac{1}{2}\right)-\frac{v^{2}}{2}+\frac{1}{2 v^{2}}\left(m[m+1]+\frac{1}{4}\right),
$$


where $v$ is the quantum number of the 1D harmonic oscillator. Note that this spectrum preserves the degeneracy of the Kramers pairs, and in particular there are two ground states with the energy

$$
E_{00}=E_{0-1}=\frac{1}{2}-\frac{v^{2}}{2}+\frac{1}{8 v^{2}} .
$$

This spectrum has been shown to match the strong-SOC spectrum for the low-energy states very well $[41,45]$. In this approximation, momentum-state wave functions are

$$
\Psi_{v m}=f_{v}(p-v) \frac{1}{\sqrt{2 p}}\left(\begin{array}{c}
-i e^{-i \theta} \\
1
\end{array}\right) \frac{e^{-i m \theta}}{\sqrt{2 \pi}},
$$

where

$$
f_{v}(p)=\frac{1}{\sqrt{2^{v} v !}} \frac{1}{\pi^{1 / 4}} e^{-p^{2} / 2} H_{v}(p)
$$

are the 1D harmonic oscillator eigenstates and $H_{v}$ denotes the Hermite polynomial.

We now apply the translation operator in the $x$ direction on the two ground states,

$$
\begin{aligned}
& T \Psi_{00}=\Psi_{00}-\frac{i x_{0}}{2}\left(\frac{\Psi_{11}}{\sqrt{2}}+\frac{\Psi_{1-1}}{\sqrt{2}}+v \Psi_{01}+v \Psi_{0-1}\right), \\
& T \Psi_{0-1}=\Psi_{0-1}-\frac{i x_{0}}{2}\left(\frac{\Psi_{10}}{\sqrt{2}}+\frac{\Psi_{1-2}}{\sqrt{2}}+v \Psi_{00}+v \Psi_{0-2}\right),
\end{aligned}
$$

where we have used one of the Hermite function recursion relations, namely,

$$
p f_{n}(p)=\sqrt{\frac{n}{2}} f_{n-1}(p)+\sqrt{\frac{n+1}{2}} f_{n+1}(p) .
$$

Subsequently, Eqs. (13) and (15) yield

$$
\begin{gathered}
\langle x\rangle=\frac{x_{0}}{4}\left(\cos t+2 \cos \frac{t}{v^{2}}+\cos \left[t+\frac{t}{v^{2}}\right]\right), \\
\left\langle y \sigma_{z}\right\rangle=-\frac{x_{0}}{4} \sin \frac{t}{v^{2}} .
\end{gathered}
$$

Taking a Fourier transform of the $\langle x\rangle$ signal, discarding very small frequencies, and restoring dimensions yield two peaks at the energies $\hbar \omega_{T}$ and $\hbar \omega_{T}+1 / M v^{2}$. We compare these analytical results with a numerical simulation for $v=10$ in Fig. 3. Note that in this strong SOC regime, center-of-mass oscillations are qualitatively different from the dipole mode in the absence of SOC. In particular, here the center-of-mass position oscillates around the initial position $x_{0}$ and not the bottom of the trap [46], which is ultimately due to the physical momentum being substantially different from the canonical momentum [64]. Note further that the amplitude of the spin-dipole response is only one quarter of that of center-of-mass oscillations.

\section{EXACT NUMERICAL RESULTS}

While at zero temperature for small perturbation amplitude $x_{0} \ll 1$ and extreme SOC strengths $v \ll 1$ and $v \gg 1$ we have managed to obtain analytical results, other regimes remain unexplored. To address this, we turn to numerically evaluating
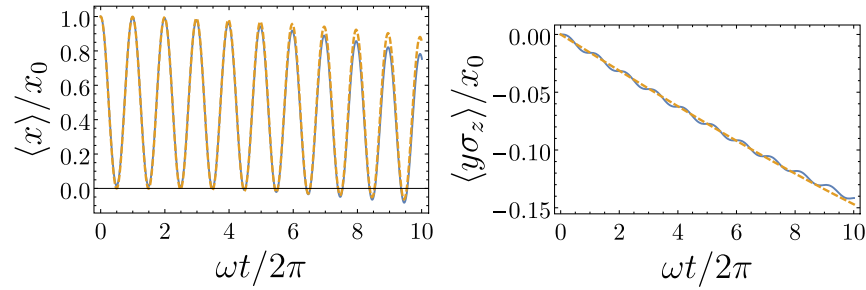

FIG. 3. Center-of-mass position and spin-dipole moment oscillations (spin Hall mode) as a response to displacement of the two ground states along the $x$ direction with the displacement amplitude $x_{0}$ in the strong SOC regime $(v=10)$. We compare exact numerical results ( $x_{0}=1 / 100$, blue solid line; see Sec. IV for more details) to the analytical results in Eqs. (36) and (37) (infinitesimal $x_{0}$, yellow dashed line) for ten trap periods. Fourier transforming the $\langle x\rangle$ signal, discarding very small frequencies, and restoring dimensions yield two peaks at the energies $\hbar \omega_{T}$ and $\hbar \omega_{T}+1 / M v^{2}$.

the evolution of the system. Noninfinitesimal translation amplitudes are taken into account by employing the displaced number states [65]. In this way, we can cover perturbation amplitudes and SOC strengths of any magnitude. We consider, moreover, nonzero temperature.

We now investigate the amplitude of the spin-dipole moment oscillations in response to a small (one tenth of the trap length) displacement of the center-of-mass position of a system of either bosons or fermions. We limit the time of evolution to ten trap periods to account for the finite lifetime of ultracold atomic samples in the experiment. In order to explore qualitative effects of statistics and temperature on the spin Hall mode, we consider a system of 100 particles at three temperatures, $k_{B} T=1 / 10,1,3 / 2$ in the units of trap energy, where $k_{B}$ is the Boltzmann constant. The number of particles is limited by computational requirements.

In practice, we numerically diagonalize the Hamiltonian to obtain the spectrum and the eigenstates. We fix the number of particles $N=100$ and solve

$$
N=\sum_{s} \frac{1}{\exp \left(\left[E_{s}-\mu\right] / k_{B} T\right) \mp 1}
$$

for the chemical potential $\mu$, where the sum runs over the eigenstate energies $E_{s}$ and the upper (lower) sign corresponds to bosonic (fermionic) statistics. Subsequently, we compute how the expectation values evolve in time:

$$
\begin{gathered}
\langle x\rangle=\frac{1}{N} \sum_{s} \frac{\langle x\rangle_{s}}{\exp \left(\left[E_{s}-\mu\right] / k_{B} T\right) \mp 1}, \\
\left\langle y \sigma_{z}\right\rangle=\frac{1}{N} \sum_{s} \frac{\left\langle y \sigma_{z}\right\rangle_{s}}{\exp \left(\left[E_{s}-\mu\right] / k_{B} T\right) \mp 1},
\end{gathered}
$$

where the time-dependent center-of-mass position and spindipole moment for a state $s$, namely, $\langle x\rangle_{s}$ and $\left\langle y \sigma_{z}\right\rangle_{s}$, have been defined in Eqs. (12) and (14). We emphasize that these quantities are evaluated exactly in our noninteracting system [66]. Since the results for bosons and fermions are quite different, we present them one after another.

For bosons the amplitude of the response is given in Fig. 4. For all investigated temperatures, the plot can be roughly divided into three parts: weak, strong, and intermediate SOC 


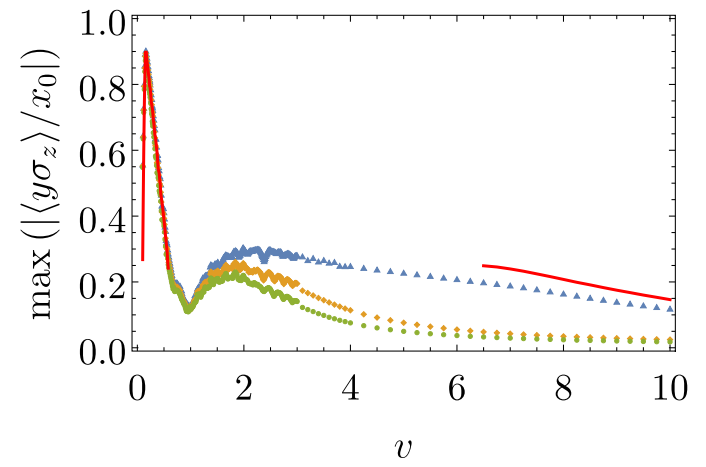

FIG. 4. Maximum amplitude of the transverse spin-dipole moment $\left\langle y \sigma_{z}\right\rangle$ for 100 bosons, normalized to the initial center-of-mass displacement amplitude $x_{0}$ (equal to $1 / 10$ of the trap length), observed over the time interval of ten trap periods. Three temperatures in the units of the trap energy have been investigated: $k_{B} T=0.1$ (blue triangles), 1 (orange diamonds), and 1.5 (green circles). At weak and strong SOC, the response can be described analytically at zero temperature [red solid curves, Eqs. (41) and (37); see Sec. IV for more details].

strength. Whereas the explanation of the first two regimes is relatively straightforward, the same cannot be said about the intermediate regime of $0.6 \lesssim v \lesssim 7$. In the limit of weak SOC (small $v$ ), the evolution of the spin-dipole moment displays a very weak temperature dependence and can be described analytically (Sec. III A). This approximation is accurate only up to the first peak, which occurs at $v \simeq 0.15$. However, by employing Eq. (20) as an ansatz we are able to fit the response up to $v \simeq 0.6$. Explicitly, we use

$$
\left\langle y \sigma_{z}\right\rangle=-a(v) \cos t \sin f(v) t,
$$

where now the amplitude $a$ and the frequency $f$ depend on the SOC strength (see Fig. 5, left). For small $v$, the response is virtually independent of temperature in the range that we have investigated, as almost exclusively the two degenerate ground states are occupied. This is because the spectrum in this regime consists of weakly perturbed harmonic oscillator states, therefore resulting in exponentially suppressed occupation of excited states for bosons. We are also able to explain the results in the limit of strong SOC $v \gtrsim 7$ at low temperature, as there the spin-dipole moment approximately follows the
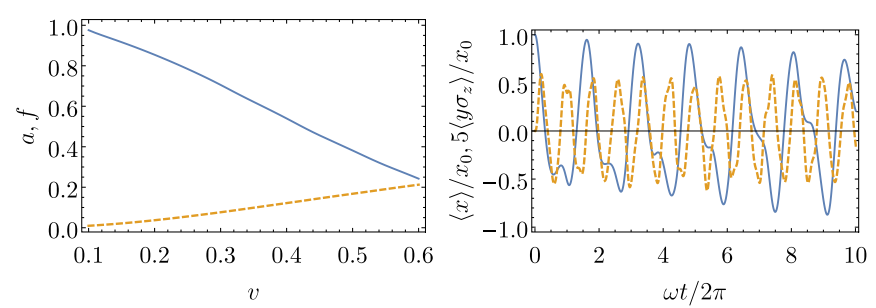

FIG. 5. Left: the amplitude $a$ (blue solid line) and the frequency $f$ (yellow dashed line) fits for the spin-dipole moment ansatz in Eq. (41) of 100 bosons. Right: the center-of-mass position (blue solid line) and the transverse spin-dipole moment (yellow dashed line; multiplied by a factor of 5 for clarity) for 100 bosons at $v=1$ (intermediate SOC strength), where the amplitude of the spin-dipole moment response shows a local minimum for bosonic particles at all considered temperatures. evolution described in Eq. (37). However, in this regime the match is not perfect at any nonzero temperature, and it worsens as the system is heated. This is because the gaps in the spectrum decrease for moderate SOC strength due to effective dimensional reduction [see Eq. (29)], and thus, excited states are readily occupied. Note that this is also the reason why the effect of temperature on the response is the strongest for moderately strong SOC.

To reiterate, in the strong $(v \gg 1)$ SOC regime, Eq. (26) holds; that is, the wave function is accurately described by $\psi_{-}$. That is, even though there are two branches of states in the system originating from the two dispersion branches in the absence of a trap, the upper branch is empty, as the energy gap between these two branches is large. Furthermore, in this regime, $p \simeq v$ is a good approximation, and also the last term in the dispersion in Eq. (29) becomes small. The latter means the degeneracy of the lowest-energy manifold becomes large. Thus, either bosons or fermions primarily occupy this lowest-energy $v=0$ manifold. However, the gaps between the manifolds are finite (equal to 1 in our units), and thus, higher-energy manifolds can also be thermally occupied, suppressing the response as temperature increases. This is to be contrasted with the moderate-SOC-strength situation, where the $1 / v^{2}$ term in the dispersion cannot be neglected. Therefore, the lowest-energy manifold degeneracy is less pronounced, and thus, the occupation of a large number of states is strongly dependent on temperature. This in turn explains the strong dependence of the response on temperature for moderate SOC strength in Fig. 4.

When several states are occupied, the behavior becomes more complicated. In particular, since different-energy states contribute oscillations of different frequencies and phases to the response, it is not straightforward to explain the minimum and the maximum shown in Fig. 4 for the intermediate values of $v$. To illustrate the typical response in this intermediate regime, in Fig. 5 (right) we show the evolution of the transverse spin-dipole moment for $v=1$, which is the local response minimum for all considered temperatures.

Occupation of several states is particularly relevant for fermions (Fig. 6). In that case, the interference of responses of various states plays a crucial role, resulting in several local minima and maxima, which become sharper as temperature decreases. However, the qualitative structure of the response is similar to bosons, in the sense that there is a global maximum at $v \simeq 0.13$ (compare to $v \simeq 0.15$ for bosons), and the response becomes progressively weaker for stronger SOC. In the region $3<v<10$ the response is below 0.05 and decays very slowly with increasing SOC strength for all considered temperatures, and hence, we have excluded this region from the plot.

All the results reported thus far are in the limit of small initial displacement. The response of the system is nonlinear in the sense that the maximum amplitude of the spin-dipole moment oscillation strongly depends on the initial displacement (Fig. 7). Furthermore, we again emphasize that the dynamics described often follows the collapse and revival type of behavior due to many states involved. Therefore, in order to investigate the effects of various parameters on the response (such as SOC strength, center-of-mass displacement amplitude, and temperature), it is important to keep the observation time constant. 


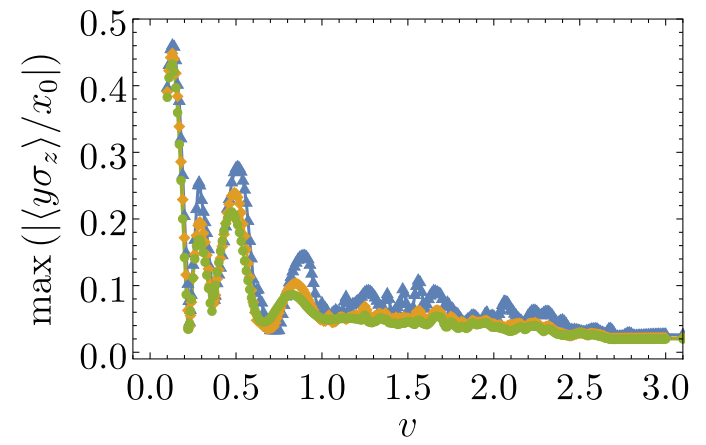

FIG. 6. Maximum amplitude of the transverse spin-dipole moment $\left\langle y \sigma_{z}\right\rangle$ for 100 fermions, normalized to the initial center-of-mass displacement amplitude $x_{0}$ (equal to $1 / 10$ of the trap length), observed over the time interval of ten trap periods. Three temperatures in the units of the trap energy have been investigated: $k_{B} T=0.1$ (blue triangles), 1 (orange diamonds), and 1.5 (green circles). The points at each temperature are joined by a line of the corresponding color for clarity.

\section{SUMMARY AND OUTLOOK}

We have investigated a harmonically trapped system with Rashba SOC and no interparticle interactions. In particular, we have studied the response of the system to a small displacement away from the bottom of the trap. We found that in addition to the expected center-of-mass oscillations, a dynamics of the spin-dipole moment is induced. This spin-dipole moment dynamics is transverse to the displacement direction and is analogous to the spin Hall effect. Therefore, we dubbed this collective mode the spin Hall mode.

Furthermore, we have performed an exact numerical study of the qualitative effects of temperature and statistics on the amplitude of the spin-dipole moment oscillations. For bosons in the weak-SOC and strong-SOC limits, the response is captured by analytic expressions. In the intermediate-SOC region for bosons, as well as for fermions at any SOC strength, the spin-dipole moment oscillations appear anharmonic. Even though the amplitude of these oscillations as a function of the SOC strength is different for bosons and fermions, we have found that in regions where the spin Hall response is the strongest, the effects of temperature and statistics are weak. We hope that our analysis will stimulate experimental work on collective modes of ultracold atomic gases with 2D SOC.

In future work, building on more formal results [67], it might be possible to extend the simple analytical treatment

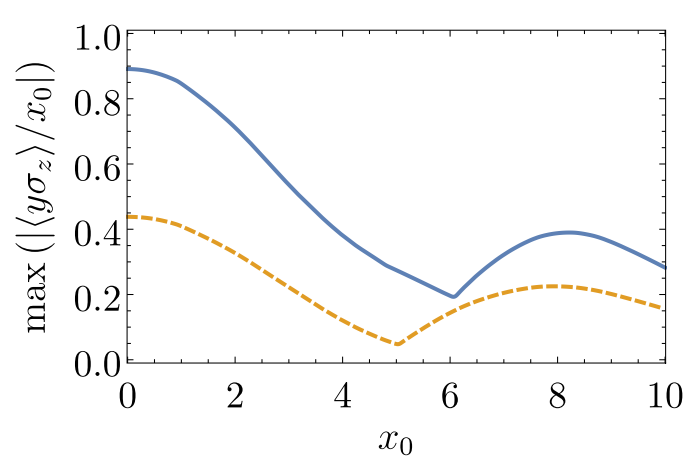

FIG. 7. Maximum normalized amplitude of the transverse spindipole moment $\left\langle y \sigma_{z}\right\rangle$ for 100 bosons (solid blue line) and fermions (dashed yellow line) at a temperature of $k_{B} T=1 / 10$ and SOC strength $v=0.15$ observed over the time interval of ten trap periods as a function of the initial center-of-mass displacement amplitude $x_{0}$.

presented here. Furthermore, as some of the realistic Rashba SOC implementation schemes [33] might result in various anisotropies, it is both feasible [54-56] and desirable to investigate the effects of such anisotropies on the spin Hall mode in an approach similar to the one presented here. In order to make quantitative predictions for experiments, in addition to anisotropies, careful accounting of the nonlinear behavior of the system is important. Exploring interaction effects, for example, in a mean-field (Hartree-Fock) type of treatment is another promising research direction. Finally, one could investigate how the results presented here are altered in a Bose-Einstein-condensed phase or in the presence of pair condensation.

\section{ACKNOWLEDGMENTS}

It is our pleasure to thank D. Trypogeorgos, I. Spielman, L. Pitaevskii, O. Marchukov, and S. Stringari for stimulating discussions. J.A. has received funding from the European Union's Horizon 2020 research and innovation program under the Marie Skłodowska-Curie Grant Agreement No. 706839 (SPINSOCS). R.A.D. and H.T.C.S. are supported by the Stichting voor Fundamenteel Onderzoek der Materie (FOM) and are part of the D-ITP consortium, a program of the Netherlands Organisation for Scientific Research (NWO) that is funded by the Dutch Ministry of Education, Culture and Science (OCW). R.A.D. is also supported by the European Research Council (ERC).
[1] D. W. Preston and E. R. Dietz, The Art of Experimental Physics (Wiley, New York, 1991).

[2] D. Shoenberg, Magnetic Oscillations in Metals (Cambridge University Press, Cambridge, 1984).

[3] Springer Handbook of Acoustics, edited by T. D. Rossing (Springer, Dordrecht, 2014).

[4] L. Levitov and G. Falkovich, Nat. Phys. 12, 672 (2016).

[5] D. A. Bandurin, I. Torre, R. K. Kumar, M. Ben Shalom, A. Tomadin, A. Principi, G. H. Auton, E. Khestanova, K. S.
Novoselov, I. V. Grigorieva, L. A. Ponomarenko, A. K. Geim, and M. Polini, Science 351, 1055 (2016).

[6] J. O. Andersen, Rev. Mod. Phys. 76, 599 (2004).

[7] S. Giorgini, L. P. Pitaevskii, and S. Stringari, Rev. Mod. Phys. 80, 1215 (2008).

[8] W. Kohn, Phys. Rev. 123, 1242 (1961).

[9] R. Bamler and A. Rosch, Phys. Rev. A 91, 063604 (2015).

[10] J. Dalibard, F. Gerbier, G. Juzeliūnas, and P. Öhberg, Rev. Mod. Phys. 83, 1523 (2011). 
[11] N. Goldman, G. Juzeliūnas, P. Öhberg, and I. B. Spielman, Rep. Prog. Phys. 77, 126401 (2014).

[12] Y.-C. Zhang, Z.-Q. Yu, T. K. Ng, S. Zhang, L. Pitaevskii, and S. Stringari, Phys. Rev. A 94, 033635 (2016).

[13] S. Stringari, Phys. Rev. Lett. 118, 145302 (2017).

[14] J.-Y. Zhang, S.-C. Ji, Z. Chen, L. Zhang, Z.-D. Du, B. Yan, G.-S. Pan, B. Zhao, Y.-J. Deng, H. Zhai, S. Chen, and J.-W. Pan, Phys. Rev. Lett. 109, 115301 (2012).

[15] Z. Chen and H. Zhai, Phys. Rev. A 86, 041604 (2012).

[16] D. S. Hall, M. R. Matthews, J. R. Ensher, C. E. Wieman, and E. A. Cornell, Phys. Rev. Lett. 81, 1539 (1998).

[17] G. Modugno, M. Modugno, F. Riboli, G. Roati, and M. Inguscio, Phys. Rev. Lett. 89, 190404 (2002).

[18] S. B. Koller, A. Groot, P. C. Bons, R. A. Duine, H. T. C. Stoof, and P. van der Straten, New J. Phys. 17, 113026 (2015).

[19] T. Bienaimé, E. Fava, G. Colzi, C. Mordini, S. Serafini, C. Qu, S. Stringari, G. Lamporesi, and G. Ferrari, Phys. Rev. A 94, 063652 (2016).

[20] B. DeMarco and D. S. Jin, Phys. Rev. Lett. 88, 040405 (2002).

[21] G. Valtolina, F. Scazza, A. Amico, A. Burchianti, A. Recati, T. Enss, M. Inguscio, M. Zaccanti, and G. Roati, Nat. Phys. 13, 704 (2017).

[22] I. Ferrier-Barbut, M. Delehaye, S. Laurent, A. T. Grier, M. Pierce, B. S. Rem, F. Chevy, and C. Salomon, Science 345, 1035 (2014).

[23] M. Delehaye, S. Laurent, I. Ferrier-Barbut, S. Jin, F. Chevy, and C. Salomon, Phys. Rev. Lett. 115, 265303 (2015).

[24] R. Roy, A. Green, R. Bowler, and S. Gupta, Phys. Rev. Lett. 118, 055301 (2017).

[25] J. Sinova, D. Culcer, Q. Niu, N. A. Sinitsyn, T. Jungwirth, and A. H. MacDonald, Phys. Rev. Lett. 92, 126603 (2004).

[26] J. Sinova, S. O. Valenzuela, J. Wunderlich, C. H. Back, and T. Jungwirth, Rev. Mod. Phys. 87, 1213 (2015).

[27] A. Manchon, H. C. Koo, J. Nitta, S. M. Frolov, and R. A. Duine, Nat. Mater. 14, 871 (2015).

[28] G. Dresselhaus, Phys. Rev. 100, 580 (1955).

[29] J. Armaitis, J. Ruseckas, and G. Juzeliūnas, Phys. Rev. A 95, 033635 (2017).

[30] M. C. Beeler, R. A. Williams, K. Jiménez-García, L. J. LeBlanc, A. R. Perry, and I. B. Spielman, Nature (London) 498, 201 (2013).

[31] C. J. Kennedy, G. A. Siviloglou, H. Miyake, W. C. Burton, and W. Ketterle, Phys. Rev. Lett. 111, 225301 (2013).

[32] J. D. Sau, R. Sensarma, S. Powell, I. B. Spielman, and S. Das Sarma, Phys. Rev. B 83, 140510 (2011).

[33] D. L. Campbell, G. Juzeliūnas, and I. B. Spielman, Phys. Rev. A 84, 025602 (2011).

[34] B. M. Anderson, I. B. Spielman, and G. Juzeliūnas, Phys. Rev. Lett. 111, 125301 (2013).

[35] D. L. Campbell and I. B. Spielman, New J. Phys. 18, 033035 (2016).

[36] L. Huang, Z. Meng, P. Wang, P. Peng, S.-L. Zhang, L. Chen, D. Li, Q. Zhou, and J. Zhang, Nat. Phys. 12, 540 (2016).
[37] A. Eckardt, Rev. Mod. Phys. 89, 011004 (2017).

[38] C. Chin, R. Grimm, P. Julienne, and E. Tiesinga, Rev. Mod. Phys. 82, 1225 (2010).

[39] E. van der Bijl and R. A. Duine, Phys. Rev. Lett. 107, 195302 (2011).

[40] J. Larson and E. Sjöqvist, Phys. Rev. A 79, 043627 (2009).

[41] S. Sinha, R. Nath, and L. Santos, Phys. Rev. Lett. 107, 270401 (2011).

[42] C. Wang, C. Gao, C.-M. Jian, and H. Zhai, Phys. Rev. Lett. 105, 160403 (2010).

[43] S.-K. Yip, Phys. Rev. A 83, 043616 (2011).

[44] H. Hu, B. Ramachandhran, H. Pu, and X.-J. Liu, Phys. Rev. Lett. 108, 010402 (2012).

[45] H. Hu and X.-J. Liu, Phys. Rev. A 85, 013619 (2012).

[46] Y. Zhang, L. Mao, and C. Zhang, Phys. Rev. Lett. 108, 035302 (2012).

[47] Z.-Q. Yu and H. Zhai, Phys. Rev. Lett. 107, 195305 (2011).

[48] X. Y. Yin, S. Gopalakrishnan, and D. Blume, Phys. Rev. A 89, 033606 (2014).

[49] J. Radić, S. S. Natu, and V. Galitski, Phys. Rev. Lett. 112, 095302 (2014).

[50] C. D. Schillaci and T. C. Luu, Phys. Rev. A 91, 043606 (2015).

[51] S. Mardonov, M. Modugno, and E. Y. Sherman, J. Phys. B 48, 115302 (2015).

[52] E. Doko, A. L. Subaş1, and M. Iskin, Phys. Rev. A 93, 033640 (2016).

[53] E. Doko, A. L. Subaş1, and M. Iskin, Phys. Rev. A 95, 013601 (2017).

[54] O. V. Marchukov, A. G. Volosniev, D. V. Fedorov, A. S. Jensen, and N. T. Zinner, J. Phys. B 46, 134012 (2013).

[55] O. V. Marchukov, A. G. Volosniev, D. V. Fedorov, A. S. Jensen, and N. T. Zinner, J. Phys. B 47, 195303 (2014).

[56] O. V. Marchukov, D. V. Fedorov, A. S. Jensen, A. G. Volosniev, and N. T. Zinner, Eur. Phys. J. D 69, 73 (2015).

[57] S. S. Natu and S. Das Sarma, Phys. Rev. A 88, 033613 (2013).

[58] J. Larson, B. M. Anderson, and A. Altland, Phys. Rev. A 87, 013624 (2013).

[59] I. B. Bersuker, The Jahn-Teller Effect (Cambridge University Press, Cambridge, 2006).

[60] J. Larson, Phys. Rev. A 81, 051803 (2010).

[61] J. Larson, E. N. Ghassemi, and A. Larson, Europhys. Lett. 101, 43001 (2013).

[62] D. M. Stamper-Kurn and M. Ueda, Rev. Mod. Phys. 85, 1191 (2013).

[63] X.-L. Qi and S.-C. Zhang, Rev. Mod. Phys. 83, 1057 (2011).

[64] J. Armaitis, J. Ruseckas, and E. Anisimovas, Phys. Rev. A 95, 043616 (2017).

[65] K. E. Cahill and R. J. Glauber, Phys. Rev. 177, 1857 (1969).

[66] The code required to compute the spectrum and the time evolution of the center-of-mass position and the spin-dipole moment has been made available online: https://github.com/jogundas/zaltys.

[67] H. Tütüncüler, R. Koç, and E. Olğar, J. Phys. A 37, 11431 (2004). 